\title{
Guidelines enforcement and clinical outcome
}

\author{
Donato Santovito - Leopoldo Di Iorio - Chiara Mammarella • \\ Enza Di Lembo · Camilla Paganelli • Andrea Mezzetti • \\ Francesco Cipollone
}

Received: 31 October 2012/ Accepted: 21 November 2012/Published online: 22 January 2013

(C) SIMI 2013

\section{Case presentation}

Dr. Di Iorio, Dr. Mammarella, Dr. Di Lembo

A.F., a 61-year-old man, was admitted to our department for severe and persistent fever [body temperature(BT) $40.0^{\circ} \mathrm{C}$ ] with chills, associated with sore throat and cough. The patient had a history of arterial hypertension, mixed dyslipidemia and hyperuricemia, treated respectively with ramipril plus hydrochlorothiazide, rosuvastatin, $n$-PUFAs and allopurinol. Ten years before admission, he underwent cardiac surgery with aortic valve replacement (mechanical prosthesis Sorin Carbomedics Orbis 25) for severe valvular stenosis. Six years later he again underwent cardiovascular surgery for a thoracic aortic aneurysm with implantation of a prosthetic tube (Vascutek-28). After these procedures, he received permanent oral anticoagulation (warfarin).

Blood tests performed at hospital admission showed ESR $102 \mathrm{~mm} / \mathrm{h}$, neutrophilic leukocytosis, hypoalbuminemia with polyclonal hypergammaglobulinemia, and severe hypoxaemia with hypercapnia. Blood and urine cultures were obtained (several consecutive collections performed before the start of antibiotic therapy), chest X-ray study, 12-lead electrocardiogram (ECG), abdominal and pelvic ultrasonography, pharyngeal tampons, and the analysis of antiviral antibodies showed no clinically significant

D. Santovito and L. Di Iorio contributed equally to this article.

D. Santovito - L. Di Iorio - C. Mammarella - E. Di Lembo .

C. Paganelli · A. Mezzetti · F. Cipollone ( $\square)$

European Center of Excellence on Atherosclerosis, Hypertension and Dyslipidemia, "G. d'Annunzio" University, Via dei Vestini, 66100 Chieti, Italy

e-mail: fcipollone@unich.it findings. Despite these results, considering the life-threatening condition, we decided to start oxygen therapy and antibiotic therapy. During the first days of hospitalization, the patient continued to show severe intermittent fever (B.T. $\max 40.5^{\circ} \mathrm{C}$ ), despite the introduction of two consecutive i.v. broad spectrum antibiotic therapies (initially ceftriaxone, followed by piperacillin plus tazobactam i.v.).

\section{Dr. Santovito, Dr. Paganelli}

On the basis of clinical presentation and the patient's medical history (valve and aortic prostheses), we posited the presence of an acute infective endocarditis (IE). With respect to this clinical situation, the current American Heart Association/American College of Cardiology (AHA/ ACC) [1] as well as the more recent European Society of Cardiology (ESC) [2] guidelines recommend transesophageal echocardiography (TEE) as a first-line diagnostic tool in patients with a prosthetic valve (Class I; level of evidence $\mathrm{C}$ ) to confirm the diagnosis. In our case, however, despite the high clinical suggestion of IE, the consulting cardiologist decided to perform a transthoracic echocardiogram, following a personal and empiric cost-effectiveness and time-consuming evaluation. Unfortunately, this procedure failed to demonstrate any pathological alteration in valve structure or function.

\section{Dr. Di Iorio, Dr. Mammarella, Dr. Di Lembo}

A few days after admission to the hospital, the patient complained of an intense headache, and was found to have a left homonymous hemianopsia and a dreamy state. An immediate brain CT scan documented an acute intracranial haemorrhage. It was located in an atypical site (right occipital lobe), was characterized by abnormal morphology 
(multilobular), and was complicated by perilesional edema with mass effect. Due to the atypical localization, we would have liked to perform a brain magnetic resonance (MRI) to clarify the nature of this brain lesion (rupture of an arteriovenous malformation? haemorrhagic transformation of an embolic infarction? mycotic aneurysm break?). Unfortunately, the presence of epicardial electrodes, residual from the previous procedure of valve replacement, did not permit this study.

Consultant neurosurgeons according to the site and dimension of the hemorrhagic lesion (occipital lobe, axial diameter $3.4 \mathrm{~cm}$ ) and to the condition of the patient (Glasgow Coma Scale 15, unaltered state of consciousness, no clinical signs of endocranial hypertension) excluded the need to perform an urgent surgical procedure, and suggested a conservative (medical) approach. After the decision for a medical rather than surgical strategy, we initially supported the patient's blood haemostasis by vitamin $\mathrm{K}$ (the original PT-INR value was 3.1 ), and then replaced warfarin with low molecular weight heparin (despite the presence of an acute brain hemorrhage, there was nevertheless the persistence of a high thrombotic risk associated with the presence of a mechanical valve, which we deemed essential for undertaking a persistent anticoagulant therapy). In addition, the patient received a blood transfusion as the haemoglobin value dropped to $6.6 \mathrm{~g} / \mathrm{dl}$ from the original value of $11.8 \mathrm{~g} / \mathrm{dl}$ observed at the admission. Multiple factors could explain this severe anemia (persistent severe inflammation, bleeding in the presence of anticoagulant therapy, acute brain haemorrhage, stress ulcer of the upper gastrointestinal tract).

\section{Dr. Santovito, Dr. Paganelli}

The presence of sudden intracranial haemorrhage further increases the clinical probability of IE. In fact, intracranial haemorrages are included in the disease's vascular phenomena, and represent minor criteria for the diagnosis of endocarditis according to the modified Duke criteria [3]. According to these criteria, our patient had a "possible" diagnosis of IE because three distinct minor criteria were concomitantly expressed, namely, predisposing cardiac conditions, fever, and intracranial haemorrhage. Thus, in the presence of such a high clinical probability, we submitted a new request for a second echocardiographic evaluation, specifically by TEE. In fact, according to the AHA/ACC [1] and ESC guidelines [2], in patients symptomatic and with high clinical suggestion of IE, even if the TTE did not show cardiac abnormalities, a TEE should be performed. Conversely, again a new TTE was performed, and again failed to demonstrate any clinical valvular abnormality.
Dr. Di Iorio, Dr. Mammarella, Dr. Di Lembo

In the following days, there was an additional evolution of the clinical scenario. The patient manifested arthralgias and symmetric adjunctive polyarthritis involving the hands and knees [radio-ulno-carpal, metacarpal-phalangeal, proximal and distal interphalangeal and patello-femoral joints, (Fig. 1), right-sided lumbar pain, purpurae and petechiae on both the lower and upper extremities (Fig. 2)]. In the initial differential diagnosis, these findings were considered to be either signs of septic embolization due to acute endocarditis, or as skin lesions in the context of a systemic autoimmune vasculitis.

A subsequent abdominal ultrasonography revealed an anechoic image adjacent to the right kidney. This image was not present in the initial ultrasonographic examination. A subsequent abdominal CT scan confirmed the liquid nature of this peri-renal image, and suggested the diagnosis of haematoma (probably the hemorrhagic evolution of an embolic infarction) and extended caudally to the

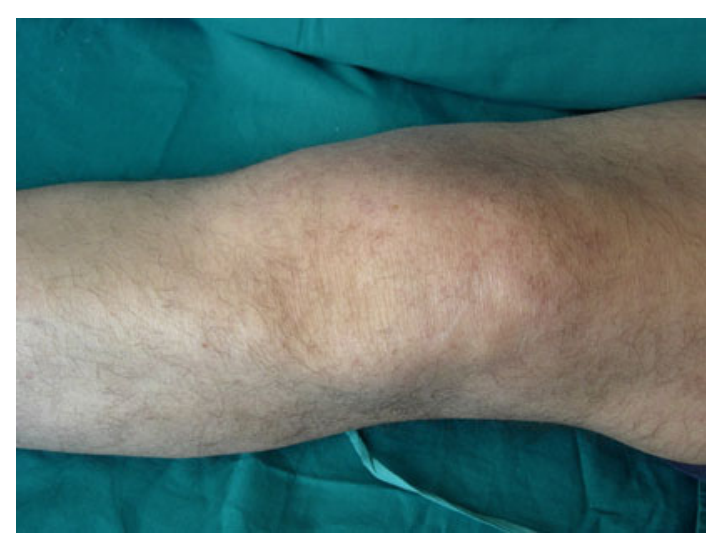

Fig. 1 Acute arthritis involving the left patello-femoral joint. Note that the joint presents all the classical signs of acute inflammation: pain, heat, redness, tumefaction and loss of function

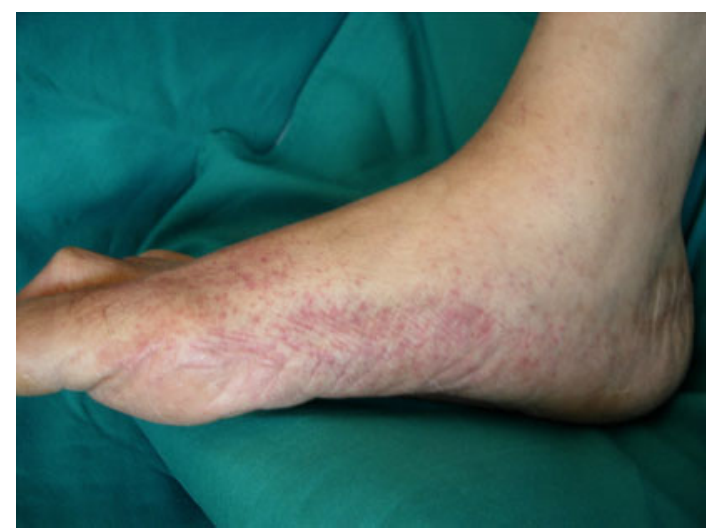

Fig. 2 Purpura and petechiae of the internal surface of the right foot, due to microembolization of small cutanous vessels 
retroperitoneum, along the homolateral psoas muscle. New blood tests showed the persistence of severe systemic inflammation (ESR $76 \mathrm{~mm} / \mathrm{hs}$, CRP $13.4 \mathrm{mg} / \mathrm{dl}$ ), associated with hypoalbuminemia, serum complement consumption, microscopic hematuria, and proteinuria in a non-nephrotic range. Immunologic tests (ANA, ENA, ANCA, cryoglobulins), and markers of hepatitis, performed in order to exclude the presence of a systemic vasculitis, were all negative. The ECG, originally devoid of pathological abnormalities, now showed a ventricular bigeminy with several polymorphic ectopic beats.

\section{Clinical diagnosis}

\section{Dr. Cipollone, Dr. Mezzetti}

The clinical pattern (history of prosthesic valve and aortic replacement, persistent septic fever with chills, continuous arterial embolization, petechiae, neurologic manifestations, embolic focal glomerulonephritis) and the laboratory findings (anemia, leukocytosis, elevated erythrocyte sedimentation rate and C-reactive protein) strongly supported the diagnosis of acute IE. Unfortunately, all the blood cultures were negative; however, it is well known that up to $31 \%$ of all patients with acute IE have negative blood cultures [4], often as a consequence of prior (at home) antibiotic therapy. Based on these considerations, and despite two negative transthoracic echocardiograms, finally, a TEE was performed. This examination revealed, at the site of the aortic valve, the presence of a large perivalvular fistulizing abscess. Subsequently an angio-CT scan confirmed the diagnosis, and also underlined the extension of the infective process up to the mediastinic aditus (Fig. 3). Thus, we added to the initial antibiotic therapy (i.v. piperacillin plus tazobactam) a full dose of i.v. vancomycin (a specific treatment for methicillin-resistant Staphylococcus aureus) and immediately

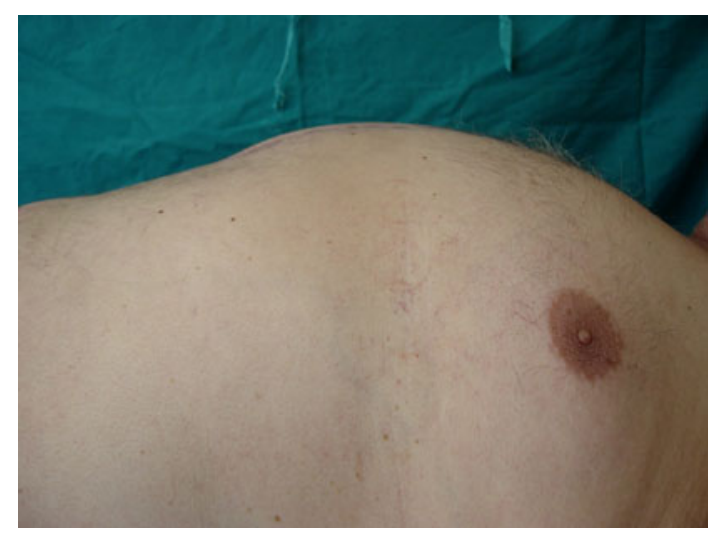

Fig. 3 Podgy thorax, due to the extension of the infectious process into mediastinum transferred the patient to the cardiac surgery unit for an immediate intervention of valve replacement and perilesional curettage.

\section{Final diagnosis}

Acute infective endocarditis complicated by perivalvular and periaortic abscess and mediastinitis.

\section{Discussion}

\section{Dr. Cipollone, Dr. Santovito, Dr. Mezzetti}

IE is an infection of the endocardial surface of the heart including heart valves $[2,5]$. In western countries, IE usually affects older patients as the result of healthcare-associated procedures (i.e. dental procedures), especially in patients with a predisposing factor such as prosthetic valve replacement $[6,7]$. An early diagnosis is important for a clinical successful treatment. Echocardiography is a powerful diagnostic tool to make the diagnosis of IE, and should be performed as soon as possible. However, in case of high clinical probability, especially in a patient with a prosthetic valve, a negative transthoracic echocardiography cannot definitively exclude the presence of IE, and immediate TEE is recommended as a first-line diagnostic approach $[1,2]$. In fact, even in patients with native valve endocarditis, transthoracic echocardiography cannot detect vegetations $<2 \mathrm{~mm}$ in diameter, and can correctly identify only $25 \%$ of vegetations $<5 \mathrm{~mm}$ and $70 \%$ of those between 6 and $10 \mathrm{~mm}$ [8]. In addition, in $20 \%$ of all patients this study is technically inadequate because of emphysema or body habitus. Thus, transthoracic echography may generally detect vegetations in 40-63\% of all patients with definite clinical IE (even with recent advances in technologies, although this might provide better results in the near future [9]), and therefore the overall sensitivity of this technique is low. Moreover, transthoracic echocardiography is not adequate for evaluating prosthetic valves (sensitivity for prosthetic valve endocarditis is as low as $20 \%$ ) or for detecting intracardiac complications [8]. On the contrary, TEE is safe, and significantly more sensitive than transthoracic echocardiography. In fact, it may detect vegetations in $>90 \%$ of patients with definite endocarditis. Therefore, TEE is the gold standard study for making the diagnosis of prosthetic valve endocarditis, or for the detection of myocardial abscess, valve perforation, and intracardiac fistulae. Nevertheless, the diagnosis of IE can never be excluded simply by negative echocardiogram findings, either transthoracic or transesophageal. In cases like this, the adherence to current evidence-based guidelines (i.e. early use of TEE in patients 
with suspected acute prosthetic valve endocarditis), surely would result in a more rapid diagnosis and in the immediate start of appropriate antimicrobial treatment.

According to recent reports, Staphylococci (both Staphylococcus aureus, $30 \%$, and coagulase-negative Staphylococcus $22 \%$ ) have emerged as the most common infecting microorganisms in prosthetic valve endocarditis $[10,11]$. Thus, vancomycin and gentamicin are both recommended as first-line treatment $[2,12]$. Rifampicin can penetrate the biofilm of the most common infecting microorganisms on the prosthetic valves, and should therefore be added to vancomycin or gentamicin, particularly in IE occurring within the first 12 months after valve implantation. Surgery is required in $25-50 \%$ of cases of acute IE; however, this indication is not supported by strong clinical evidence (such as multicentric randomized trials), and the clinical decision as well as the timing for the surgical intervention should be tailored on the basis of the individual benefit/risk ratio [13]. Usually, in cases complicated by intracranial haemorrhage, which correlates with a worse neurological prognosis, surgery must be postponed for at least 1 month [2, 14]. However, in the case of our patient, the extensive perivalvular diffusion of the infection despite massive antibiotic therapy represented an absolute indication for immediate (within days) surgical intervention.

\section{Conclusion}

\section{Dr. Mammarella, Dr. Cipollone}

This is an interesting and complex clinical case regarding a man admitted to our observation for fever of unknown origin who had a final diagnosis of acute infectious endocarditis. One point of interest of this report is that the patient did not receive an immediate diagnosis despite a suggestive medical history, clinical presentation and clinical course, as a consequence of not following current guidelines. The patient developed many complications of IE, possibly due to delayed diagnosis. In conclusion, this episode suggests that a full knowledge and implementation of current evidence based guidelines are necessary for rapid identification of IE, early treatment initiation, reduction of cardiac and extracardiac complications, and improvement of a patient's outcome.

\section{Conflict of interest None.}

\section{References}

1. American College of Cardiology, American Heart Association Task Force on Practice Guidelines (Writing Committee to revise the 1998 guidelines for the management of patients with valvular heart disease), Society of Cardiovascular Anesthesiologists, Bonow RO, Carabello BA, Chatterjee K, de Leon AC, Faxon DP, Freed MD, Gaasch WH, Lytle BW, Nishimura RA, O'Gara PT, O'Rourke RA, Otto CM, Shah PM, Shanewise JS, Smith SC, Jacobs AK, Adams CD, Anderson JL, Antman EM, Fuster V, Halperin JL, Hiratzka LF, Hunt SA, Lytle BW, Nishimura R, Page RL, Riegel B (2006) ACC/AHA 2006 Guidelines for the management of patients with valvular heart disease: a report of the American College of Cardiology/American Heart Association Task Force on Practice Guidelines (writing Committee to Revise the 1998 guidelines for the management of patients with valvular heart disease) developed in collaboration with the Society of Cardiovascular Anesthesiologists endorsed by the Society for Cardiovascular Angiography and Interventions and the Society of Thoracic Surgeons. J Am Coll Cardiol 48:e1-148

2. Habib G, Hoen B, Tornos P, Thuny F, Prendergast B, Vilacosta I, Moreillon P, de Jesus Antunes M, Thilen U, Lekakis J, Lengyel M, Muller L, Naber CK, Nihoyannopoulos P, Moritz A, Zamorano JL, ESC Committee for Practice Guidelines CPG, Vahanian A, Auricchio A, Bax J, Ceconi C, Dean V, Filippatos G, Funck-Brentano C, Hobbs R, Kearney P, McDonagh T, McGregor K, Popescu BA, Reiner Z, Sechtem U, Sirnes PA, Tendera M, Vardas P, Widimsky P, Document Reviewers, Vahanian A, Aguilar R, Bongiorni MG, Borger M, Butchart E, Danchin N, Delahaye F, Erbel R, Franzen D, Gould K, Hall R, Hassager C, Kjeldsen K, McManus R, Miro JM, Mokracek A, Rosenhek R, San Roman Calvar JA, Seferovic P, Selton-Suty C, Uva MS, Trinchero R, van Camp G (2009) Guidelines on the prevention, diagnosis, and treatment of infective endocarditis (new version 2009): The Task Force on the Prevention, Diagnosis, and Treatment of Infective Endocarditis of the European Society of Cardiology (ESC). Eur Heart J 30:2369-2413

3. Li JS, Sexton DJ, Mick N, Nettles R, Fowler VG, Ryan T, Bashore T, Corey GR (2000) Proposed modifications to the Duke criteria for the diagnosis of infective endocarditis. Clin Infect Dis 30:633-638

4. Lamas CC, Eykyn SJ (2003) Blood culture negative endocarditis: analysis of 63 cases presenting over 25 years. Heart 89:258-262

5. Mylonakis E, Calderwood SB (2001) Infective endocarditis in adults. N Engl J Med 345:1318-1330

6. Hill EE, Herijgers P, Claus P, Vanderschueren S, Herregods M-C, Peetermans WE (2007) Infective endocarditis: changing epidemiology and predictors of 6-month mortality: a prospective cohort study. Eur Heart J 28:196-203

7. Murdoch DR, Corey GR, Hoen B, Miró JM, Fowler VG, Bayer AS, Karchmer AW, Olaison L, Pappas PA, Moreillon P, Chambers ST, Chu VH, Falcó V, Holland DJ, Jones P, Klein JL, Raymond NJ, Read KM, Tripodi MF, Utili R, Wang A, Woods CW, Cabell CH (2009) International Collaboration on Endocarditis-Prospective Cohort Study (ICE-PCS) Investigators. Clinical presentation, etiology, and outcome of infective endocarditis in the 21st century: the International Collaboration on EndocarditisProspective Cohort Study. Arch Intern Med 169:463-473

8. Evangelista A, Gonzalez-Alujas MT (2004) Echocardiography in infective endocarditis. Heart 90:614-617

9. Casella F, Rana B, Casazza G, Bhan A, Kapetanakis S, Omigie J, Reiken J, Monaghan MJ (2009) The potential impact of contemporary transthoracic echocardiography on the management of patients with native valve endocarditis: a comparison with transesophageal echocardiography. Echocardiography. 26:900-906

10. Wang A, Athan E, Pappas PA, Fowler VG, Olaison L, Paré C, Almirante B, Muñoz P, Rizzi M, Naber C, Logar M, Tattevin P, Iarussi DL, Selton-Suty C, Jones SB, Casabé J, Morris A, Corey GR, Cabell CH (2007) International Collaboration on Endocarditis-Prospective Cohort Study Investigators. Contemporary 
clinical profile and outcome of prosthetic valve endocarditis. JAMA 297:1354-1361

11. Lee JH, Burner KD, Fealey ME, Edwards WD, Tazelaar HD, Orszulak TA, Wright AJ, Baddour LM (2011) Prosthetic valve endocarditis: clinicopathological correlates in 122 surgical specimens from 116 patients (1985-2004). Cardiovasc Pathol 20:26-35

12. Gould FK, Denning DW, Elliott TSJ, Foweraker J, Perry JD, Prendergast BD, Sandoe JAT, Spry MJ, Watkin RW (2012) Working Party of the British Society for Antimicrobial
Chemotherapy. Guidelines for the diagnosis and antibiotic treatment of endocarditis in adults: a report of the Working Party of the British Society for Antimicrobial Chemotherapy. J Antimicrob Chemother 67:269-289

13. Prendergast BD, Tornos P (2010) Surgery for infective endocarditis: who and when? Circulation 121:1141-1152

14. Eishi K, Kawazoe K, Kuriyama Y, Kitoh Y, Kawashima Y, Omae $T$ (1995) Surgical management of infective endocarditis associated with cerebral complications. Multi-center retrospective study in Japan. J Thorac Cardiovasc Surg 110:1745-1755 